\title{
Transposição de grandes artérias: a importância do teste do coraçãozinho como exame de triagem
}

\author{
Transposition of large arteries: the importance of the little heart test as a screening test
}

Transposición de arterias grandes: la importancia de la pequeña prueba del corazón como prueba de detección

José Rodolfo Bogiani Bearare ${ }^{1 *}$, Luiz Felipe Bottura Napolitano1, Rafael Akio Watanabe Nakakogue ${ }^{1}$, Kelly Luisa Cintra ${ }^{1}$.

\section{RESUMO}

\begin{abstract}
Objetivo: Relatar o caso de um paciente que teve como resultado do teste do coraçãozinho falso negativo. Detalhamentos De Caso: Ao nascimento, paciente apresentou bom estado geral de saúde de acordo com a triagem obrigatória do hospital, na qual recebeu alta hospitalar. Com 14 dias de vida, apresentou cianose central, saturação $70 \%$ e hiperfluxo pulmonar, sendo diagnosticado com transposição de grandes artérias. Aos 30 dias de vida, realizou-se cirurgia profilática, optando-se pela técnica de Mustard/Senning. Em 2018, foi identificado fibrilação atrial em consulta com cardiologista pediátrica sendo provável consequência da dilatação atrial direita, controlada por medicação, associada a sopro sistólico/diastólico. Em 2020, paciente se encontra em bom estado geral e encontra-se apto para atividades físicas recreativas e de educação física. Considerações Finais: Paciente se encontrar saudável atualmente, embora terá maior probabilidade de iniciar um quadro de insuficiência cardíaca por déficit inotrópico em decorrência da técnica cirúrgica adotada, a qual foi consequência do errôneo resultado do teste do coraçãozinho.
\end{abstract}

Palavras-chave: Cardiopatias, Diagnóstico tardio, Cirurgia torácica.

\section{ABSTRACT}

Objective: Report a patient case with false negative heart test result. Case Details: At birth, patient presented good general health according to mandatory hospital screening, in which he has hospital discharged. At 14 days of age, he presented central cyanosis, $70 \%$ saturation and pulmonary hiperflow, being diagnosed with transposition of large arteries. At 30 days of life, corrective surgery was performed, opting for Mustard/Senning technique. In 2018, it was identified atrial fibrillation in consultation with a pediatric cardiologist, with a probable consequence of medication-controlled right atrial dilation associated with systolic/diastolic murmur. In 2020, patient is in good general condition and is fit for recreational physical activities and physical education. Final Considerations: Actualmente el paciente está sano, aunque es más probable que comience con insuficiencia cardíaca debido a un déficit inotrópico debido a la técnica quirúrgica adoptada, que fue consecuencia del resultado erróneo de la pequeña prueba cardíaca.

Keywords: Heart diseases, Delayed diagnosis, Thoracic surgery.

\section{RESUMEN}

Objetivo: Informar el caso de un paciente que tuvo el resultado de la prueba cardíaca falsa negativa. Detalles Del Caso: Al nacer, el paciente tenía un buen estado general de salud de acuerdo de la detección obligatoria en el hospital, en el que fue dado de alta. A los 14 días de edad, presentó cianosis central, 70\% de saturación y edema pulmonar, siendo diagnosticado con transposición de arterias grandes. A los 30 días de vida, se realizó una cirugía correctiva, optando por la técnica de Mustard/Senning. En 2018, la fibrilación auricular se identificó en consulta con un cardiólogo pediátrico, lo que probablemente sea consecuencia de la dilatación de la aurícula derecha, controlada por medicamentos, asociada con soplo sistólico/diastólico. En 2020, el paciente se encuentra en buen estado general y es apto para actividades físicas recreativas y educación física. Consideraciones Finales: Actualmente el paciente está sano, aunque es más probable que comience con insuficiencia cardíaca debido a un déficit inotrópico debido a la técnica quirúrgica adoptada, que fue consecuencia del resultado erróneo de la pequeña prueba cardíaca.

Palabras clave: Cardiopatías, Diagnóstico tardío, Cirugía torácica.

\footnotetext{
${ }^{1}$ Universidade de Franca (UNIFRAN), Franca - SP. *E-mail: rodolfo.bilac@hotmail.com
} 


\section{INTRODUÇÃO}

Cardiopatias congênitas são defeitos anatômicos da bomba cardíaca decorrentes do processo da má formação embrionária do órgão, afetando aproximadamente $1 \%$ dos nascidos vivos variando a incidência por exemplo, de acordo com síndromes genéticas. Essa condição pode gerar graves desconfortos e levar ao óbito, tendo como principais fatores extragenéticos o uso de medicações inadequadas devido a diagnósticos e condutas médicas, tendo o Teste do Coraçãozinho a importância de garantir a triagem de cardiopatias congênitas potencialmente fatais. (PENHA JG, et al., 2015; SANTANA MVT, 2014).

A Dextro-Transposição das Grandes Artérias (D-TGA) é a cardiopatia congênitacianogênica mais freqüente ao nascimento, incidindo entre $0,218-0,442 / 1.000$ nascidos vivos, reconhecida pela primeira vez por Baillie M (1797). No D-TGA, a aorta origina-se do ventrículo direito e a artéria pulmonar tem origem do ventrículo esquerdo, resultando em circulações pulmonares e sistêmicas independentes (ATIK E e MOREIRA VM, 2011). A TGA estabelece dois circuitos fechados, incompatíveis, e que se não houver comunicação entre eles, seja no nível atrial, ventricular ou arterial, o paciente irá a óbito (WARNES CA, 2006).

Para obter o diagnóstico de D-TGA, a equipe deve suspeitar quando o recém-nascido apresenta cianose central desde o nascimento ou nos primeiros dias de vida, devido à queda da resistência vascular pulmonar, que piora nas mamadas ou quando a criança chora. Outros sintomas, como hipoxemia grave e acidose, dependerão da magnitude do fluxo pulmonar, da mistura de sangue e de suas consequências. Quando o recém-nascido com D-TGA não apresenta esses sintomas precocemente é porque deve haver alguma outra anomalia associada, o que dificulta o diagnóstico (DEMETER CN, et al., 2019).

Alguns estudos demonstram que se não houver diagnóstico precoce e tratamento cirúrgico, $28.7 \%$ das crianças podem evoluir para o óbito na primeira semana de vida, $51.6 \%$ no primeiro mês e $89.3 \%$ não sobrevivem até o primeiro ano (STOUT KK, et al., 2016). Cardiopatia congênita não diagnosticada pode apresentar complicações posteriores, levando à readmissão no hospital, instabilidade hemodinâmica ou, até mesmo, estado de choque que evolui para óbito nos primeiros dias/semanas de vida (GOSSE SD, et al., 2017).

A avaliação do recém-nascido com a medição, não invasiva, da saturação de oxigênio entre 24 e 48 horas de vida é conhecida como o teste do coraçãozinho. Este teste consiste na aferição da oximetria de pulso (quanto oxigênio o sangue está transportando) por meio de um oxímetro (equipamento essencial para monitorar pacientes em hospitais e maternidades) como detecção precoce de bebês que apresentam malformações cardiovasculares, em especial D-TGA por ser uma condição com alto índice de mortalidade. A oximetria de pulso é um método de fácil acesso e disponibilidade; sua precisão torna este dispositivo capaz de identificar hipoxemia de difícil detecção via avaliação clínica nas primeiras 24 horas de vida, mostrando ser um método de triagem neonatal capaz de salvar vidas de recém-nascidos com cardiopatias congênitas (MAWSON IE, et al., 2018).

O teste do coraçãozinho é um método simples, indolor, não invasivo, rápido e de baixo custo, capaz de identificar distúrbios precocemente e permitir conduta imediata (BRASIL, 2014). Antes de sua introdução como triagem neonatal, 70 a 100 bebês morriam a cada ano nos Estados Unidos por terem diagnóstico tardio, impossibilitando tratamento correto a tempo. O teste do coraçãozinho permite que seu diagnóstico seja possível traçar possíveis patologias além de alterações cardíacas (e.g. infecções e patologias pulmonares/respiratórias) devendo ser realizada na Unidade Neonatal (CHAMSI-PASHA MA e CHAMSIPASHA H, 2016).

Considerando que as cardiopatias congênitas são anomalias na qual seu diagnóstico precoce e conduta médica correta irá determinar a condições de vida e de desenvolvimento da criança, há necessidade de melhorar a prática clínica desse profissional, para que possa desenvolver cuidados seguros e baseado em evidências científicas. Frente a isso, o objetivo deste artigo é fazer um relato de caso de paciente com transposição de grandes artérias, que teve teste do coraçãozinho falso negativo. 


\section{DETALHAMENTO DO CASO}

Trata-se de um relato de caso, descritivo e retrospectivo, em que foi realizada a análise de todos os prontuários eletrônicos de um paciente, sexo masculino, nascido em 2015 em um hospital público de uma cidade no interior do estado de São Paulo.

Buscando zelar pela integridade ética do paciente envolvido e de seus familiares, foi elaborado um Termo de Consentimento Livre e Esclarecido, na qual a análise dos prontuários só foi realizada após assinatura do responsável pela criança.

Foram analisados todos os prontuários do paciente até o presente estudo, bem como a análise de prontuário do ambulatório de cardiologia pediátrica em que o paciente é atualmente acompanhado. Nos prontuários, foi analisado sobre o momento do nascimento (dados do paciente e intercorrências), tratamentos e métodos cirúrgicos feitos no paciente e acompanhamento do seu estado de saúde geral até o momento de escrita final deste estudo (dezembro de 2019). Nos prontuários do ambulatório de cardiologia pediátrica, foi analisado resultados de exames, avaliação física do paciente e medicamentos prescritos.

Os resultados foram analisados e apresentados de maneira descritiva, com enfoque nos principais dados relacionados à saúde do paciente e sua condição cardíaca. Paciente nasceu em 22 de abril de 2015, com 38 semanas e 1 dia e peso de $2.955 \mathrm{~g}$, em um hospital público de uma cidade no interior do estado de São Paulo, parto cesáreo devido à miomatose uterina da mãe (tumores uterinos benignos de natureza muscular lisa).

Segundo dados presentes no prontuário hospitalar do paciente, mãe não apresentou riscos na gestação e, no momento do nascimento, não apresentou intercorrências. Paciente permaneceu internado por dois dias no hospital, somente para cumprir protocolo de nascimento com parto cesáreo e sem intercorrências.

Recebeu alta hospitalar devido a bom estado geral de saúde e por não apresentar riscos e alertas em virtudes dos resultados de exames e acompanhamento padrão para nascidos vivos em hospital (i.e. incluindo o teste do coraçãozinho).

Com 14 dias de vida, paciente apresentou cianose central durante amamentação, encaminhado para o hospital do nascimento, onde foi observado saturação de oxigênio de $70 \%$. Paciente ficou internado por dois dias, com quadro de hiperfluxo pulmonar o qual evoluiu com infecção de foco pulmonar, suspeitada e conduzida como pneumonia hospitalar.

Neste momento, devido a avaliação do corpo médico e ecodopplercardiograma, paciente foi diagnosticado com transposição de grandes artérias. Visto este quadro, paciente foi encaminhado para outro hospital, em outra cidade próxima a cidade em que nasceu, que possuí devida capacitação para corrigir as desordens que paciente apresentava. Devido à infecção hospitalar, foi recomendado aguardar melhora do quadro para prosseguir para uma abordagem cirúrgica que contemplava a correção da transposição de grandes artérias por técnica de Adib Jatene.

Com 30 dias de vida, foi necessário que paciente realizasse a cirurgia de correção, mesmo com a presença de infecção. Pela idade de paciente (30 dias), foi indicado cirurgia de correção com técnica Senning. Após a cirurgia, paciente permaneceu no hospital por 14 dias, com alta hospitalar assintomático. Devido a sua condição de transposição de grandes artérias, paciente iniciou uso de medicação enalapril e amiodarona com melhora parcial do quadro, que foramsubstituídos por uso contínuo de AAS 70mg/dia, carvedilol 1,56mg 12/12 horas e furosemida, otimizando assim o tratamento.

Em 2018, paciente foi encaminhado ao hospital onde nasceu, para o ambulatório de alto risco. Como acompanhamento padrão da transposição de grandes artérias, foi realizado exame Holter na qual foi evidenciado fibrilação atrial, com tratamento medicamentoso iniciado desde então.

No presente momento de escrita deste artigo, paciente apresenta assintomático, bom estado geral de saúde e apto para atividades físicas recreativas e de educação física estando hoje em condição estável, frequência cardíaca condizente com a idade e sopro 3+/6+ mêsossistólico em todos os focos com maior audibilidade em foco pulmonar. 


\section{DISCUSSÃO}

Esse artigo demonstrou o acompanhamento do estado de saúde de uma criança que recebeu o diagnóstico tardio de transposição de grandes artérias. 50 anos atrás, 30\% das crianças diagnosticadas com cardiopatias congênitas conseguiam sobreviver até a vida adulta.

Hoje, com a evolução da cirúrgica cardíaca e à adaptação do cateterismo cardíaco para recém-nascidos, além das melhorias em unidades de terapia intensiva neonatal e pediátrica, a mortalidade caiu drasticamente, com expectativa que até $85 \%$ destes recém-natos sobrevivam à vida adulta (FIXLER DE, et al., 2014). Além disso, estudos mostram que quanto mais tardia a assistência em centro de referência, maior a mortalidade (JENKINS K, 2012; FIXLER DE, et al., 2014; MOZAFFARIAN D, et al., 2016).

Os países que organizaram sua rede de assistência, seguindo este padrão de evolução, conseguiram aumentar consideravelmente a sobrevida com qualidade de vida para crianças com cardiopatias congênitas (MOZAFFARIAN D, et al., 2016).

No entanto, ainda existe alta taxa de letalidade das cardiopatias e esta demonstra que deve-se atentar a estruturação da rede de assistência especializada, à fim de atender adequadamente o volume de neonatos com cardiopatia congênita, além de prover investimentos reais em treinamento e tecnologia assistencial, ainda dentro da faixa etária neonatal visando diagnóstico precoce (LOPES SAVA, et al., 2018). Nesse estudo caso, foi observado que nos dados do prontuário de nascimento, o paciente apresentava um bom estado geral de saúde no momento da alta hospitalar, após seu nascimento. O diagnóstico tardio da transposição de grandes artérias demonstra duas hipóteses: a primeira é a possibilidade de que o hospital do nascimento apresentou erros no registro do prontuário. Um recente estudo analisou os erros nas prescrições médicas de uma Unidade de Pronto Atendimento, no qual analisaram 1.798 prescrições (MOURA SNC, et al., 2019).

Foi evidenciado que mais de $40 \%$ das prescrições apresentavam ausência ou nome incompleto do paciente e ausência de identificação do leito, além de até $20 \%$ sem a assinatura de um profissional médico, má caligrafia e prescrições sem dosagem.Erros cometidos por profissionais da rede médica podem causar danos ao paciente, colocando em risco a eficácia do tratamento, da avaliação e a própria vida do paciente (MOURA SNC, et al., 2019).

A segunda hipótese é a não realização ou realização inadequada do teste do coraçãozinho. Profissionais da área de saúde devem priorizar e adotar boas práticas para execução de procedimentos, entretanto, com a sobrecarga de trabalho e o índice de estresse intra-hospitalar, a má execução torna-se um fator de risco ao paciente, uma vez que pode ocasionar erros de diagnóstico e procedimentos (OLIVEIRA FBM, et al., 2016).

Em um hospital público, apesar dos profissionais médicos apresentarem altos níveis em relação a realização profissional, nota-se que existe a presença de exaustão profissional, sendo necessário planejamento do serviço, como aprimoramento do trabalho em equipe, que possam favorecer a qualidade dos serviços públicos e prevenir o estresse laboral, evitando erros (AMARIZ AA, et al., 2016).

Lopes SAVA, et al. (2018) identificaram que recém-nascidos prematuros, com baixo peso e presença de comorbidades apresentaram maior risco de mortalidade relacionada às cardiopatias congênitas. Apesar disso, o diagnóstico tardio da transposição de grandes artérias e o uso da técnica de Mustard/Senning, é recomendado que o diagnóstico seja realizado na primeira semana de vida, e que a adequada correção cirúrgica seja rápida (DEMETER CN, et al., 2019). A técnica mais adequada para essa cardiopatia é a de Janete, no entanto, pelo diagnóstico tardio, a melhor técnica foi a Mustard/Senning, representando mais um possível determinante para a sobrevida do paciente.

O quadro de saúde do paciente mostra-se saudável e estável, no entanto a disfunção ventricular é a complicação mais comum em pacientes com transposição de grandes artérias operados pela técnica de Senning (SANTANA MVT, 2014).

Neste contexto, é importante a criação de estratégias com intuito de promover a educação em saúde, gerando um melhor entendimento e acolhimento sobre o contexto de saúde do bebê com cardiopatias congênitas em suas mães, como por exemplo, ouvi-las. Estes achados sinalizam para a necessidade de maior investimento em tecnologia assistencial em populações com este perfil (CESARIO MSA, et al., 2020). 
Apesar de paciente estar saudável atualmente, o diagnóstico tardio da dextro-transposição de grandes artérias, decorrente do teste do coraçãozinho não ter apontado a cardiopatia, paciente terá maior chance de evoluir para insuficiência cardíaca por déficit inotrópico.

Esse artigo ressalva a importância de realizar os exames e anamnese do recém-nascido com adequada qualificação profissional e seguindo o protocolo padrão de cuidados da Sociedade Brasileira de Pediatria, com enfoque de minimizar erros de diagnóstico que poderão ser cruciais à vida da criança e sua família.

\section{REFERÊNCIAS}

1. AMARIZ AA et al. Prevalência da síndrome de burnout em médicos e médicos residentes em Montes Claros - MG no ano de 2014.Revista Unimontes Científica, 2016; 18(2):62-75.

2. ATIK E, MOREIRA VM. Imagens e correlações em cardiologia pediátrica. São Paulo: Roca, 2011.

3. BAILLIE M. The morbid anatomy of some of the most important parts of the human body. London: Johnson and Nicol, 1797.

4. BRASIL. Portaria n 20 de 10 de junho de 2014. Incorpora a oximetria de pulso, o teste do coraçãozinho a ser realizado de forma universal, fazendo parte da Triagem Neonatal do SUS. [portaria na internet]. Diário Oficial da União 11 Jun 2014 [acessado $2015 \quad$ Nov $\quad 3] . \quad$ Disponível http://bvsms.saude.gov.br/bvs/saudelegis/sctie/2014/prt0020_10_06_2014.html.

5. CESARIO MSA et al. Mães de crianças com cardiopatia congenital: dúvidas e estratégias de intervenção. Revista Eletrônica Acervo Saúde, 2020; 12(5):1-9.

6. CHAMSI-PASHA MA, CHAMSI-PASHA H. Critical congenital heart disease screening. Avicenna Journal of Medicine, 2016; 6(3):65-68.

7. DEMETER CN et al. Complex congenital heart malformation incluing transposition of the great vessels, presenting as sudden unexplained infant death. Journal of Forensic Sciences, 2019; 64(4):1241-1244.

8. FIXLER DE et al. Age at referral and mortality from critical congenital heart disease. Pediatrics, 2014; 134(1):e98-105.

9. GOSSE SD et al. CDC Grand Rounds: Newborn Screening for Hearing Loss and Critical Congenital Heart Disease. Morbidity and Mortality Weekly Report, 2017; 66: 888-890.

10. JENKINS K. Mortality with congenital heart defects in England and Wales, 1959-2009. Much progress, but more to do. Archives of Disease in Childhood, 2012; 97(10):859-60.

11. LOPES SAVA et al. Mortality for Critical Congenital Heart Diseases and Associated Risk Factors in Newborns. A Cohort Study. ArquivosBrasileiros de Cardiologia, 2018; 111(5):666-673.

12. MAWSON IE et al. Pulse oximetry findings in newborns with antenatally diagnosed congenital heart disease. European Journal of Pediatrics, 2018; 177(5):683-689.

13. MOURA SNC, et al. Análise de erros nas prescrições médicas em uma unidade de pronto atendimento do município de Juiz de Fora - MG. Revista da Escola de Ciências Médicas de Volta Redonda, 2019.

14. MOZAFFARIAN D et al. Heart disease and stroke statistics 2016 update: a report from the American Heart Association. Circulation, 2016; 133(4):e38-360.

15. OLIVEIRA FBM et al. Relationship between work overload and medication administration errors in hospital care. RevistaCiência e Saberes, 2016; 2(4).

16. PENHA JG et al. Senning paliativo no tratamento de cardiopatias congênitas com hipertensāo pulmonar grave. Arquivos Brasileiros de Cardiologia, 2015;105(4):353-361.

17. SANTANA MVT. Cardiopatias congênitas no recém-nascido: diagnóstico e tratamento. 3. ed. São Paulo: Atheneu, 2014.

18. STOUT KK et al. Chronic heart failure in congenital heart disease: a scientific statement from the American Heart Association. Circulation, 2016; 133(8):770-801.

19. WARNES CA. Transposition of the great arteries. Circulation, 2006; 114(24):2699-709. 